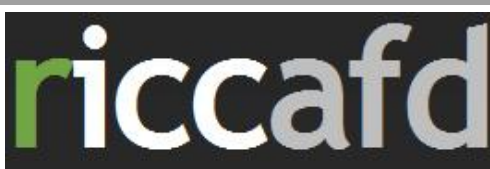

Revista Iberoamericana de Ciencias de la Actividad Física y el Deporte

\title{
EVALUACIÓN DE LA FUERZA RELATIVA DE LAS EXTREMIDADES SUPERIORES CON LA PLATAFORMA DE BOSCO
}

\section{EVALUATION OF THE RELATIVE STRENGTH OF ARMS WITH BOSCO PLATFORM}

Márquez García, F.J.

Doctor por la Universidad de Málaga (España) profesor de Educación Física en el Instituto de Enseñanza Secundaria y Bachillerato "Monterroso" de Estepona (Málaga), España. javiermarquez05@yahoo.es

Código UNESCO: 5802.99

Clasificación Consejo de Europa: 5

Recibido el 4 de abril de 2013

Aceptado el 16 de junio de 2013

PALABRAS
CLAVE:
Fuerza,
Extremidades
superiores
Rendimiento.
Plataforma de
Bosco

\section{RESUMEN}

El propósito del estudio fue comprobar si la Plataforma de contacto Ergojump, utilizada para medir la capacidad de impulsión de las extremidades inferiores a través de saltos, era válida para hacerlo sobre las extremidades superiores realizando desde tumbado prono una extensión de codos partiendo de la posición clásica de flexoextensión de brazos y antebrazos de 90 grados. Se realizó un estudio de correlación entre los registros de la plataforma $(\Phi=1)$ y un electromiógrafo. También se ha realizado análisis de la fiabilidad (e) experimental de los distintos planes de medida que conformaban las distintas variables de estudio, obteniendo óptimos resultados.

KEY WORDS:

Strength, Performance, Upper Extremities. Bosco Platform.

\section{ABSTRACT}

The purpose of the study was to test whether Ergojump contact platform, used to measure the ability of lower limb drive through jumps, was valid to do it on the upper limbs from lying prone making elbow extension starting from the classical position flexion and extension of the arms and forearms at 90 degrees. A study was conducted of correlation between records of the platform $(\Phi=1)$ and an electromyograph. Has also reliability analysis (e) experimental measure different plans that made the study variables, obtaining optimal results. 


\section{INTRODUCCIÓN}

Esta investigación pretende aumentar las posibilidades de utilización de un instrumento de medición denominado Ergojump Plus Bosco System, que se emplea habitualmente para determinar la capacidad de impulsión de las extremidades inferiores. Pensamos que podemos incrementar la validez externa de la plataforma en cuestión, aplicándola con idéntico fin para las extremidades superiores partiendo de la posición de tumbado prono. Al igual que Cogley, Teasha, Archambault, Mandy, Koverman, Youdas, y Hollman, (2005) (1), entendemos que tal gesto puede utilizarse como medio de evaluación de la fuerza de la musculatura pectoral, hombros y brazos.

En nuestro caso, analizamos el gesto de flexo-extensión de codos en su fase concéntrica adoptando una posición de partida de tumbado prono, formando un ángulo entre brazo y antebrazo de 90 grados.

No hemos hallado en la literatura científica intento alguno de llevar a cabo nuestro propósito, sin embargo sí encontramos algunas investigaciones, (Lafond 2003; Masquardt 1996; Sanders 1996) $(2,3,4)$, que pretendían aumentar la validez externa de otro tipo de plataformas o bien, comprobaban su fiabilidad y precisión.

Evidentemente, dicha evaluación sería de tipo personal debido a la diferencia de peso corporal entre sujetos, lo cual supone diferentes magnitudes de la resistencia a vencer. Ello no significa que dicho gesto no pueda ser objeto de medida de una manifestación de fuerza de un individuo; entendemos pues, que nuestro experimento se centra en el control del entrenamiento de fuerza individual del sujeto, determinando si consigue incrementos 0 decrementos en los tiempos de contacto sobre la plataforma con respecto a sí mismo en distintos momentos temporales de la planificación de la preparación física. Los aumentos de los tiempos de vuelo, suponen la aplicación de mayores niveles de fuerza, y las disminuciones están relacionadas con menores índices de dicha capacidad.

Nuestra hipótesis se basa en el precepto de que altos registros de tipo electromiográficos detectados sobre el pectoral mayor y el tríceps braquial, se corresponderán con incrementos en tiempo de contacto y altura en la flexo-extensión de codos evaluados con la plataforma.

\section{Producción y evaluación de la fuerza: las flexo-extensiones de codos.}

Hemos hallado en la literatura científica, varios estudios relevantes que implican al gesto en estudio:

Mayhew, Ball, Arnold, y Bower, (1991) (5), estudiaron la viabilidad de usar dicho gesto para predecir la ejecución concéntrica en press de banca de 1RM con 106 escolares; concluyeron que las flexoextensiones de brazos realizadas durante un minuto, no son predictores de 1RM en press de banca y por ello, no son un reflejo exacto de la fuerza de las extremidades superiores en jóvenes masculinos.

Pate, Burgess, Woods, Ross, Baumgartner (1993) (6), hallaron que las flexo-extensiones de brazos poseían validez de constructo para

Rev. lb. Cᄃ. Act.Fis. Dep. 
ser utilizados como medidas de fuerza muscular relativa al peso corporal, utilizando una muestra de niños de 9-10 años.

Invergo, Ball, y Looney (1991) (7), compararon las posibilidades de las flexo-extensiones de brazos y de la fuerza-resistencia absoluta (test en press de banca de YMCA), para predecir la máxima cantidad de peso que puede ser levantada en el ejercicio de press de banca (1RM). Los resultados mostraron que la prueba de fuerza-resistencia absoluta en press de banca fue el predictivo más efectivo para determinar la fuerza máxima (1 RM) en tal aparato.

Bentz (2003) (8), investigó sobre la ganancia de fuerza que obtendrían un grupo de alumnos de edad escolar sobre la musculatura pectoral con 8 semanas de entrenamiento a través de la ejecución de flexo-extensiones de brazos. Los resultados obtenidos mostraron que el grupo experimental consiguió una mejora de fuerza en press de banca del $12.9 \%$ y del $45.3 \%$ en flexoextensiones de brazos.

Bowersock (1999) (9), estudió la influencia antropométrica sobre la producción de potencia en el gesto de flexo-extensión de brazos. Hallaron el CDG y posteriormente realizaron el mayor número de flexo-extensiones de brazos posibles. La posición de ejecución en la que las manos están a la anchura de los hombros, mostró la correlación más fuerte con la consecución de las flexoextensiones. El análisis de regresión múltiple, mostró que la producción de potencia, peso CDG y porcentaje graso eran predictores significativos de la ejecución de flexo-extensiones de brazos. La posición de manos a la anchura de hombros, no era un predictor entre el sector masculino, pero sí lo fue con respecto a las féminas.

Murr (1997) (10), investigó la fiabilidad intersujeto e intrasujeto de dos test de flexo-extensiones de brazos: el de Fitnessgram y el Test militar (U.S.A) con una muestra fue de 100 estudiantes varones estudiantes de E.F de la Universidad de Georgia; hallaron que ambos test son válidos y fiables para medir la fuerza-resistencia de brazos y hombros en escolares masculinos.

Halem (2002) (11), estudió y propuso varias formas de ejecutar "push-ups", ya que considera el gesto de fácil ejecución y adaptable a distintos niveles de condición física. Analizó la contribución de los distintos músculos agonistas y sinergistas en el gesto según las distintas variantes que llevemos a cabo.

Hrysomallis y Kidgell (2001) (12), comprobaron si la ejecución de $5 \times 5$ $\mathrm{RM}$ en press de banca previas a la consecución de flexo-extensiones de brazos llevadas a cabo en una plataforma de fuerza, producían un aumento del impulso y de los índices de dicha capacidad; sus resultados fueron negativos.

\section{Electromiografía y actividad muscular}

Conocemos el uso de la electromiografía como medio de determinación de la acción muscular (Gouvali y Boudolos, 2005) (13). En nuestra investigación utilizamos este instrumento para comprobar que existe una

Rev. lb. Cᄃ. Act.Fis. Dep. 
correlación entre los datos obtenidos por el Ergojump Plus Bosco System y los del electromiógrafo.

Cogley, et al. (2005) (1), hicieron un estudio en el que compararon el grado de activación muscular de pectoral mayor y tríceps braquial utilizando distintas posiciones de las manos en el gesto de flexoextensiones de brazos: a la anchura de los hombros, base ancha, y base estrecha.

Los autores citan Weede y Kraemer (2002), Geiger (2004), y Donkers et al. (1993), como base conceptual de su investigación, en la que esperaban que la mayor activación muscular del tríceps tuviese lugar en la posición estrecha de manos, y la del pectoral en la ancha. Concluyen que si el objetivo es buscar la máxima activación muscular en dicho gesto, la posición de las manos debe ser estrecha.

Gouvali y Boudolos (2005) (13), pretendieron registrar los cambios dinámicos y musculares que se producían al ejecutar variantes del gesto de flexo-extensión de brazos. Para ello se valieron de una muestra de 8 sujetos con 6 variantes en la posición de los brazos en el ejercicio: normal, brazos abducidos, adducidos, posterior (con respecto a la proyección de los hombros en el suelo), anterior y con apoyo de rodillas, así como de una plataforma de fuerza para registrar las reacciones del suelo y electrodos superficiales para tomar los EMG de pectoral mayor y tríceps braquial. La carga en el momento inicial con respecto al peso corporal fue de $66.4 \%$ en la posición normal, mientras que con apoyo de rodillas suponía el $52.9 \%$. Para ambos músculos la actividad electromiográfica fue menor en la posición de apoyo de rodillas. Con el apoyo posterior, el músculo pectoral mayor fue activado más de lo normal y el tríceps, todo lo contrario.

Encontramos investigaciones electromiográficas del gesto objeto de estudio donde analizan la actividad muscular de músculos sinergistas, como las de Lear y Gross (1998) (14), quienes estudiaron las consecuencias mioeléctricas sobre la musculatura estabilizadora de la escápula (serrato anterior y parte superior e inferior del trapecio); encontraron diferencias estadísticamente significativas entre la parte alta y baja del trapecio durante la ejecución del gesto en cuestión, cuando éste se realizaba con los pies elevados respecto a las extremidades superiores.

Donkers, An, Chao, Morrey, (1993) (15), quisieron comprobar el efecto que ejercía la posición de las manos sobre la magnitud de la carga sobre la articulación del codo durante el gesto de flexo-extensiones de brazos. Observaron cómo los picos de fuerza obtenidos en el codo sobre el antebrazo suponían un promedio de un $45 \%$ del peso corporal cuando se realizaban con una anchura de brazos "normal", y disminuían cuando se hacía con una anchura menor o mayor.

Otros autores como Lehman (2005) (16), estudiaron la estimulación electromiográfica de varios músculos implicados en el gesto de flexo-extensión de codos en la posición de tumbado prono, pero ejecutados en un press de banca

Rev. Ib. CC. Act.Fis. Dep. 
con variaciones en los agarres. Concretamente analizaron la anchura del agarre en pronación y supinación sobre las porciones esternoclavicular y esternal del pectoral mayor, del bíceps braquial y el vasto lateral del tríceps braquial en un gesto isométrico sostenido. A modo de conclusión y considerando las escasas repercusiones de las distancias de agarre, la elección de la misma debe estar determinada por los requerimientos específicos del deporte para el cual se entrena.

\section{MATERIAL Y MÉTODO}

50 varones de diversa edad y niveles de condición física escogidos al azar. Todos ellos eran entrevistados previamente para comprobar si tenían algún tipo de lesión que impidiese el normal transcurso de la prueba y así descartar dicha muestra (Tabla 1).

TABLA 1: Estadísticos descriptivos de las muestras $(n=50)$

\begin{tabular}{lc}
\hline $\begin{array}{l}\text { CARACTERISTICAS } \\
\text { DE LOS SUJETOS }\end{array}$ & $\begin{array}{c}\text { Media } \pm \\
\text { Desv. } \\
\text { Típica }\end{array}$ \\
\hline $\begin{array}{l}\text { Zurdos }(\mathrm{n}=7) \\
\text { Diestros }(\mathrm{n}=43)\end{array}$ \\
EDAD $(\mathrm{años})$ & $24.14 \pm 9.82$ \\
Peso $(\mathbf{k g})$ & $72.10 \pm 10.03$ \\
Talla $(\mathbf{m})$ & $1.76 \pm .07$ \\
Envergadura $(\mathrm{m})$ & $1.77 \pm .07$ \\
IMC & $23.05 \pm 2.62$ \\
\hline
\end{tabular}

El registro de la actividad EMG se realizó mediante electrodos de superficie de $15 \mathrm{~mm}$. de diámetro. El electromiógrafo contaba con dos canales (Mioback CY-351/2), con una velocidad de lectura 30 datos/segundo entre 0.1 y 20.000 microvoltios y una frecuencia entre 30 y $300 \mathrm{~Hz}$.

Plataforma ERGO JUMP-Plus Bosco System, de rayos infrarrojos conectada a un microprocesador (Figura 1).

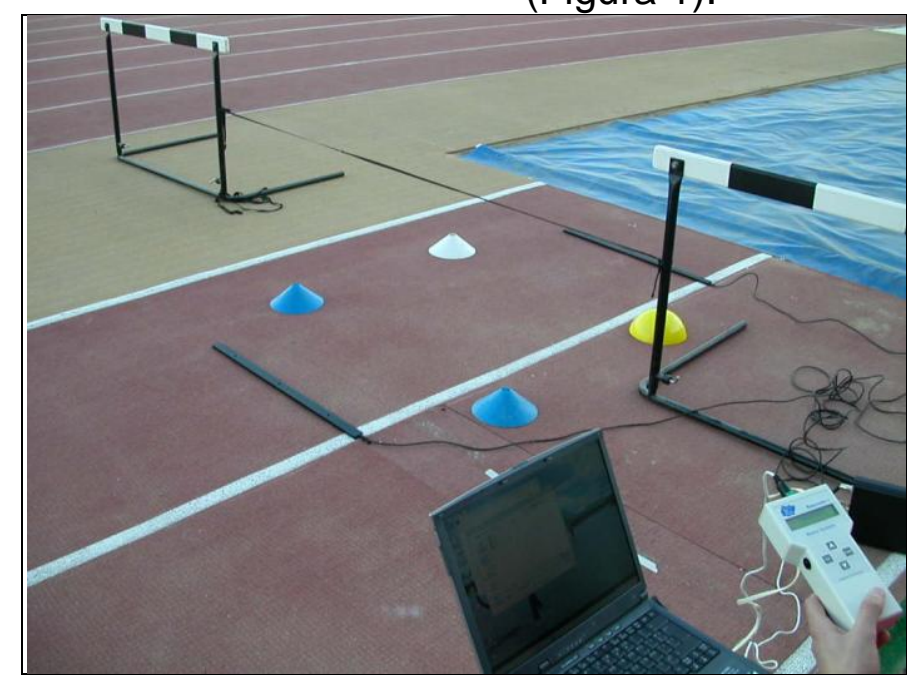

Figura 1: Ergojump-plus Bosco System

Rev. Ib. Cᄃ. Act.Fis. Dep. 
Cronómetro Decathlon Geonaute, una plomada, una escuadra, báscula Taurus Modelo Duna Typ. 3200 y Metro Cromley 34033.

Las pruebas se llevaron a cabo en el "Aula Magna" del I.E.S Monterroso de Estepona, en condiciones de temperatura de 24 grados aproximadamente, sin perjuicio del viento ni excesos de humedad.

Para la colocación de los electrodos se limpiaba la zona con alcohol, previo afeitado, situándolos al igual que hicieron Cogley et al. (2005) (1) para el caso del pectoral mayor y Gouvali et al. (2005) (13) para el tríceps braquial.

A cada sujeto, se le explicaba la ejecución del ejercicio y se le permitía una repetición a modo de prueba para comprobar que había asimilado las instrucciones dadas.

Debía situarse en cuadrupedia, con las manos dentro del área descrita por la plataforma de Bosco; con una plomada, que pendía desde los hombros, se hacía coincidir el tercer dedo de las manos con la anchura de hombros marcada por el instrumento en cuestión. A la voz de preparados, (Figura 2) habría de extender las rodillas quedando el cuerpo suspendido por dos puntos de apoyo: pies y manos. Durante tres segundos, habrían de mantenerse en flexión de codo de 90 grados comprobados con una escuadra y una vez transcurrido dicho tiempo, al llegar a "tres" (contabilización por cronómetro), habían de aplicar la máxima fuerza posible, realizando una contracción concéntrica de codos, de modo que con los brazos ya extendidos, permitiese una elevación con respecto al suelo de las manos.

El aterrizaje habría de realizarse manteniendo los codos extendidos y la amplitud de manos; la plataforma de contacto se encargaría de medir el tiempo de vuelo alcanzado y la altura.

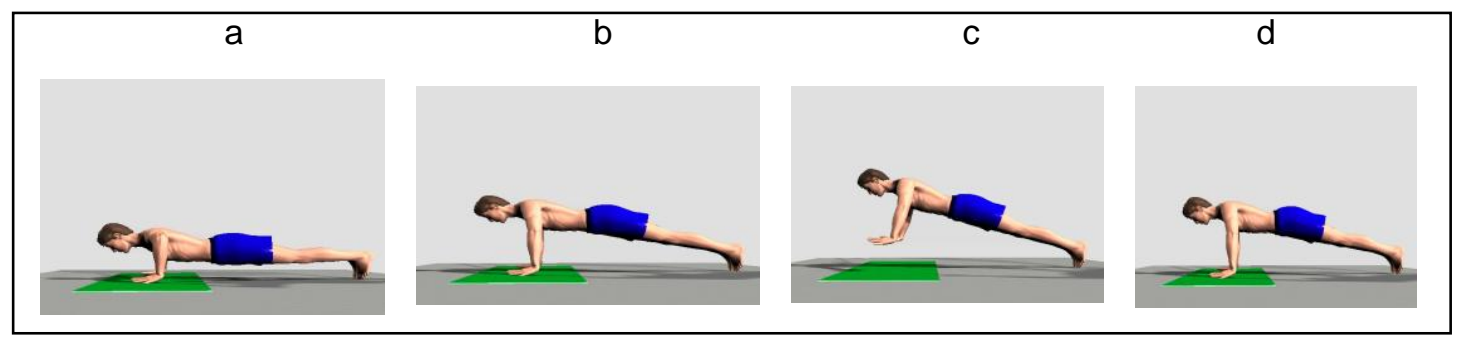

Figura 2: Ejecución de la prueba

Se realizaron dos ensayos, en los cuales se medía la respuesta electromiográfica (máximos, medias y desviaciones típicas), la altura alcanzada y el tiempo de vuelo por cada lado (izquierdo y derecho), tomándose en total cuatro medidas por sujeto. Entre ejecuciones, se permitía una pausa de 5 minutos de recuperación para evitar la fatiga muscular.

En función de la dominancia lateral del individuo, empezábamos la medición por el hemisoma correspondiente a su lado hábil y procedíamos a su registro (Figura $3)$.

Rev. Ib. CC. Act.Fis. Dep. 
Nombre:

No REGISTRO:

Teléfono:

Fecha:

Dominancia Lateral en Extremidades Superiores: D I (táłhese lo que proceda)

\begin{tabular}{|l|l|l|l|l|l|l|}
\hline & \multicolumn{3}{|l|}{ Hemisoma DERECHO } & \multicolumn{3}{l|}{ Hemisoma IZQUIERDO } \\
\hline Ensayo & Pectoral & Tríceps & Ergojump & Pectoral & Tríceps & Ergojump \\
& Mayor & Braquial & & Mayor & Braquial & \\
\hline 1 & Mx: & Mx: & Tv: & Mx: & Mx: & Tv: \\
& X: & X: & h: & X: & X: & h: \\
& Sx: & Sx: & & Sx: & Sx: & \\
\hline 2 & Mx: & Mx: & Tv: & Mx: & Mx: & Tv: \\
& X: & X: & h: & X: & X: & h: \\
& Sx: & Sx: & & Sx: & Sx: & \\
\hline
\end{tabular}

Figura 3: Hoja de registro de los datos del experimento

Posteriormente, tales registros los incluimos en una matriz en la aplicación informática SPSS 14.0 para los posteriores análisis.

Generamos otra en la que incluimos los registros electromiográficos máximos, medios, tiempos de vuelo y alturas conseguidas.

Atendiendo a la clasificación
expuesta por Hernández,
Fernández y Baptista, (2000) (21),
(Figura 4), el diseño de
investigación escogido en función
de nuestro propósito fue de tipo
transeccional (recolección de datos
de los sujetos en un único
momento) correlacional.

Atendiendo a la clasificación Fernández y Baptista, (2000) (21), (Figura 4), el diseño de investigación escogido en función de nuestro propósito fue de tipo transeccional (recolección de datos momento) correlacional.

\begin{tabular}{|l} 
Investigación Experimental: \\
$\left\{\begin{array}{l}\text { Experimento "puro": Diseño específico (de Salomón, } \\
\text { factorial, etc.). } \\
\text { Preexperimento: Diseño específico } \\
\text { Cuasiexperimento: Diseño específico }\end{array}\right.$ \\
Investigación no experimental: $\left\{\begin{array}{l}\text { Transeccional: Descriptivo. Correlacional/Causal } \\
\text { Longitudinal: De tendencia o trend. De evolución de } \\
\text { grupo o cohort. Panel. }\end{array}\right.$
\end{tabular}

Figura 4. Clasificación de los diseños de investigación (Hernández et al., 2000)

El primer paso consistió realizar el experimento con cuatro sujetos de la Facultad de Ciencias de la Educación escogidos al azar. Luego, establecimos un PLAN DE OBSERVACIÓN en el que generamos el diseño de facetas con distintos niveles, estudiándose la interacción de cada una de ellas. Utilizamos el análisis de varianza para calcular la suma de cuadrados. Pretendíamos aplicar la Teoría de la Generalizabilidad, con el fin de llevar a cabo un PLAN DE OPTIMIZACIÓN que nos determinase la amplitud de la

Rev. lb. Cᄃ. Act.Fis. Dep. 
muestra necesaria para poder generalizar los resultados obtenidos.

Dicha teoría, reconoce las múltiples fuentes de variación en los diseños de medida (individuos, observadores, sesiones, días, técnicas...) y pretende comprobar que las puntuaciones obtenidas sean representativas de un conjunto más amplio (Castellano, 2000) (17), "generalizar las observaciones reales a cualquier tipo de observaciones a las que éstas pertenezcan" (Blanco Villaseñor,
Castellano y Hernández Mendo, 2000) (19).

El análisis de generalizabilidad constituye normalmente un estudio piloto, apriorístico, que sirve para preparar una experiencia a una escala mayor (Blanco, 2001) (18), estableciéndose una serie de facetas con las que es posible determinar con precisión las fuentes de variación de cada una de ellas, y estimar el número mínimo de sesiones necesarias para generalizar con precisión cualquier resultado procedente de la investigación (Tabla 2).

TABLA 2: Porcentaje de contribución de las distintas facetas y sus interacciones en el análisis de varianza.

\begin{tabular}{|c|c|c|c|c|c|c|c|c|c|c|c|c|}
\hline $\begin{array}{l}\text { Variable } \\
\text { Dependiente }\end{array}$ & $\begin{array}{c}\text { Faceta } \\
\mathbf{S}\end{array}$ & $\begin{array}{c}\text { Faceta } \\
M\end{array}$ & $\begin{array}{c}\text { Faceta } \\
\text { SM }\end{array}$ & $\begin{array}{c}\text { Faceta } \\
\mathbf{L}\end{array}$ & $\begin{array}{c}\text { Faceta } \\
\text { SL }\end{array}$ & $\begin{array}{c}\text { Faceta } \\
\text { ML }\end{array}$ & $\begin{array}{c}\text { Faceta } \\
\mathrm{E}\end{array}$ & $\begin{array}{c}\text { Faceta } \\
\text { SE }\end{array}$ & $\begin{array}{c}\text { Faceta } \\
\text { SLE }\end{array}$ & $\begin{array}{l}\text { Faceta } \\
\text { SML }\end{array}$ & $\begin{array}{l}\text { Faceta } \\
\text { SME }\end{array}$ & $\begin{array}{l}\text { Faceta } \\
\text { SMLE }\end{array}$ \\
\hline $\begin{array}{l}\text { EMG } \\
\text { Máximos (x) }\end{array}$ & $0 \%$ & $47 \%$ & $19 \%$ & $1 \%$ & $1 \%$ & $3 \%$ & $0 \%$ & $0 \%$ & $0 \%$ & $16 \%$ & $1 \%$ & $9 \%$ \\
\hline $\begin{array}{l}\text { EMG } \\
\text { Medias (d) }\end{array}$ & $0 \%$ & $46 \%$ & $21 \%$ & $1 \%$ & $1 \%$ & $2 \%$ & $0 \%$ & $0 \%$ & $0 \%$ & $18 \%$ & $1 \%$ & $9 \%$ \\
\hline Alturas (h) & $59 \%$ & $0 \%$ & $0 \%$ & $0 \%$ & $8 \%$ & $0 \%$ & $3 \%$ & $3 \%$ & $26 \%$ & $0 \%$ & $0 \%$ & $2 \%$ \\
\hline $\begin{array}{l}\text { Tiempos de } \\
\text { vuelo }(t)\end{array}$ & $60 \%$ & $0 \%$ & $0 \%$ & $0 \%$ & $4 \%$ & $0 \%$ & $2 \%$ & $0 \%$ & $31 \%$ & $2 \%$ & $0 \%$ & $0 \%$ \\
\hline
\end{tabular}

Para ello se llevó a cabo el análisis a través de la aplicación Generalizability Study versión 2.0.E. (Ysewijn, 1996), y así concluimos que necesitábamos 48 personas consiguiendo en la práctica a 50, las cuales debían pasar por la situación experimental.

En el diseño, utilizamos un modelo de cuatro facetas: sujetos, músculo, ensayo y lado (izquierdo o derecho) con los correspondientes niveles en cada una de las variables, es decir, $50 \times 2 \times 2 \times 2$ (Tabla 3).

TABLA 3: Facetas y niveles

\begin{tabular}{cc}
\hline FACETAS & NIVELES \\
\hline Sujetos & 50 \\
Músculos & 2 (pectoral y tríceps) \\
Ensayos & 2 \\
Lado & 2 (izquierdo y derecho) \\
\hline
\end{tabular}

Establecimos una matriz que incluyesen las cuatro facetas, así como los datos de registro electromiográficos máximos, medios, y los propios de la plataforma de bosco, es decir, tiempo de vuelo y altura.

Dicha matriz fue sometida a un tratamiento informático (SAS) para

Rev. Ib. CC. Act.Fis. Dep. 
la obtención de la suma de cuadrados de las distintas facetas con respecto a las variables dependientes de tipo electromiográfica $(x, \quad d)$ y del ergojump (t, h).

\section{RESULTADOS}

Para la obtención de resultados se han utilizado las aplicaciones informáticas SAS, SPSS 14.0, y GT 2.0 (Generalizability Study. Ysewijn, 1996).

A través de la aplicación informática SAS, realizamos un análisis de varianza para obtener las sumas de cuadrados, de las distintas variables que constituirían los distintos planes de medida.

Las características descriptivas de los datos registrados con la plataforma de Bosco y el electromiógrafo están descritas en las Tablas 4 y 5.

Posteriormente, y a partir de la suma de cuadrados de las distintas facetas, procedimos al análisis de Generalizabilidad, con la aplicación informática GT en la que obtuvimos los resultados mostrados en la Tabla 7.

Con un asterisco, resaltamos los modelos de facetas que han resultado fiables (e) y generalizables $(\Phi)$ ya que los coeficientes de generalizabilidad están entre 0.9 y 1 .

Con dos asteriscos, los modelos que cuentan con una muy alta fiabilidad y generalizabilidad.

En color blanco, hallamos modelos que no son fiables y tampoco generalizables.
Podemos observar cómo la generalizabilidad del estudio es variable, mientras que la fiabilidad del mismo se mantiene en valores óptimos en los planes de medida que hemos considerado (MLE/S, SLE/M, SLM/E, SME/L), teniendo encuenta las distintas variables dependientes $(\mathrm{x}, \mathrm{d}, \mathrm{h}, \mathrm{t})$.

El objetivo fundamental de la presente investigación, es demostrar que existe una correlación entre los registros EMG con los datos (tiempo de vuelo y alturas) de la plataforma de Bosco. Para tal fin, llevamos a cabo una revisión de distintos estadísticos aplicados a estudios biosanitarios; asumimos lo expuesto por Molinero (2001) (20), quien afirma que "en la práctica de la medición de variables fisiológicas, los resultados observados, raramente mostrarán relaciones tan exactas" (es decir, de tipo lineal); por ello, dado que los registros de tipo electromiográficos, son de naturaleza biológica, hemos optado por descartar el análisis de correlación habitual, el cual establece relaciones lineales entre variables y realizamos el aconsejado por el autor: el análisis correlacional intraclase.

Para ello, con la aplicación SAS, hicimos un cálculo de la suma de cuadrados entre los modelos resultantes de dichas variables.

Una vez obtenida la suma de cuadrados, procedimos al análisis de correlación intra-clase en la aplicación GT 2.0, con las variables expuestas en la Figura 5, obteniendo los resultados expresados en la Tabla 6.

Rev. Ib. CC. Act.Fis. Dep. 
Variable electromiográfica/ Variable de la plataforma de Bosco

$\mathrm{d} / \mathrm{xh}$

$d / x t$

$\mathrm{x} / \mathrm{dh}$

$\mathrm{x} / \mathrm{dt}$

Figura 5: Variables dependientes del Electromiógrafo y del Ergojump.

Dados los coeficientes de correlación obtenidos $(\mathrm{e}=1)$, podemos afirmar que los resultados de la investigación son positivos (Pelegrina, 1999)(21), confirmándose nuestras expectativas establecidas en la hipótesis correlacional de investigación.

\section{DISCUSIÓN}

No hemos encontrado en la literatura científica situaciones experimentales

cuasiexperimentales como la que hemos planteado, aunque sí hallamos dos investigaciones donde se analiza el gesto de flexoextensión de codos y se tienen en cuenta registros electromiográficos $y$ angulaciones articulares (Lear et al., 1998; Donders et al., 1993) $(14,15)$.

Aunque en nuestro estudio no hemos analizado los mismos músculos, también hemos hallado diferencias entre el pectoral mayor y el tríceps, a favor del segundo (Tabla 4).

Tras los resultados obtenidos en el análisis de correlación intra-clase (Tabla 6) sobre las variables electromiográficas $\mathrm{d}, \mathrm{x}$ y las propias de la plataforma de Bosco $t$ y $h$, cuyos resultados mostraban una correlación positiva absoluta (e2 $=1.000$ ), así como los excelentes coeficientes de fiabilidad (Tabla 6), se confirma la hipótesis que nos habíamos planteado; podemos decir que cuando mayores registros de tiempos de vuelo se obtengan en la plataforma, mayor habrá sido la aplicación de fuerza por parte del individuo en cuestión.

Analizando los coeficientes de generalizabilidad, observamos cómo para las variables de tipo electromiográficas máximas $(x)$ y medias (d), el número de sujetos y de ensayos es suficiente para poder generalizar los resultados obtenidos ( $\Phi=0.982$ y 0.938 para las máximas y 0.980 y 0.944 para las medias respectivamente), mientras que el número de músculos $(\mathrm{m})$ y hemisomas (I) considerados, no resultaban satisfactorios.

Si tenemos en cuenta los resultados de las estimaciones de los distintos planes de medida para la Plataforma de Bosco, tomando como variable dependiente el tiempo de vuelo, el número de sujetos $\quad(\Phi=0.567), \quad$ ensayos ( $\Phi=0.786)$, y hemisomas $(\Phi=0.773)$ no han sido suficientes. En cambio, la faceta músculo $(\Phi=0.992)$, sí resulta generalizable, aunque tal registro no tiene sentido en el marco de la plataforma.

Analizando la variable dependiente altura (h), conseguimos resultados análogos, con leves variaciones en los coeficientes de generalizabilidad ( $\Phi=0.590$ para los sujetos, $\Phi=0.989$ para los músculos, $\Phi=0.780$ en el caso de lados, $\Phi=0.801$ para los ensayos).

Rev. Ib. CC. Act.Fis. Dep. 
Entendemos que los niveles de las facetas músculo y lado son fijos ya que no pueden ampliarse en el primer caso, por incapacidad del electromiógrafo de medir más grupos musculares, y en el segundo por cuestiones obvias de lateralidad diestra 0 zurda, sin más posibilidades.

Si analizamos los coeficientes de fiabilidad (e2), comprobamos cómo se obtienen resultados óptimos en todos las estimaciones de los planes de medida, lo cual es indicativo de la buena reproductibilidad de la investigación. Centrándonos en las características descriptivas de las muestras, y a pesar del amplio rango de edad (media de 24.14 años con una desviación típica de 9.82), los resultados de la investigación han sido exitosos. EI IMC medio (23.05) mostraba que contábamos con una población que se puede considerar saludable desde el punto de vista de la composición corporal. De las 50 muestras, 7 de ellos tenían una dominancia lateral izquierda y el resto de tipo derecha.

$\mathrm{Si}$ analizamos los estadísticos descriptivos correspondientes a las variables electromiográficas y de la plataforma de Bosco (Tabla 4), observamos que la media de los registros máximos para el pectoral mayor y el tríceps ha sido de 213.05 $\mathrm{mV}$ (microvoltios), mientras que la media de los registros medios ha sido de $157.12 \mathrm{mV}$.

Centrándonos en los tiempos de vuelo, el máximo alcanzado ha sido de 0.40 segundos, que se corresponde con una altura de 0.20 metros. En cualquier caso, la media de éstos ha sido de 0.24 segundos y 0.07 metros de altura.

\begin{tabular}{|c|c|}
\hline Variables & Media+Desv. Típica \\
\hline EMG máximos de pectoral y tríceps. $(\mathrm{mV})$ & $213.05 \pm 149.63$ \\
\hline EMG medias de pectoral y tríceps. (mV) & $157.12+119.26$ \\
\hline Tiempos de vuelo(s) & $.24 \pm .05$ \\
\hline Altura (m) & $.07+.03$ \\
\hline
\end{tabular}

En cuanto a las diferencias entre grupos musculares (Tabla 5), observamos al igual que Gouvali et al. (2005) (13), que existen distintos niveles de registro mioeléctrico para el pectoral mayor y el tríceps braquial. De hecho, en nuestra investigación hemos comprobado cómo los registros medios de los máximos electromiográficos, han sido mayores para el tríceps braquial que para el pectoral mayor (302.60 mV frente a $124.52 \mathrm{mV}$ ). Las medias de los registros medios han sido de $87.49 \mathrm{mV}$ para el pectoral mayor y $226.51 \mathrm{mV}$ para el tríceps braquial.

Rev. Ib. CC. Act.Fis. Dep. 
TABLA 5. Descriptivos de los resultados obtenidos en la plataforma de Bosco y variables electromiográficas de la musculatura del pectoral mayor y tríceps braquial

\begin{tabular}{lc}
\hline Variables & Media \pm Desv. Típica \\
\hline Tiempo de vuelo $(\mathrm{s})$ & $0.24 \pm 0.05$ \\
Alturas $(\mathrm{m})$ & $0.07 \pm 0.03$ \\
EMG máx pectoral mayor $(\mathrm{mV})$ & $124.52 \pm 71.90$ \\
EMG máx tríceps $(\mathrm{mV})$ & $302.60 \pm 158.51$ \\
EMG med pectoral $(\mathrm{mV})$ & $87.49 \pm 49.89$ \\
EMG med Tríceps braquial(microvoltios) & $226.51 \pm 126.92$ \\
\hline
\end{tabular}

En cualquier caso, corroboramos lo expuesto por Cogley et al. (2005), Halem (2002), (1,11), quienes estudiaron el comportamiento electromiográfico de ambos músculos según la anchura de manos adoptada durante la flexoextensión de codos de tipo concéntrica, resultando mayor la participación del tríceps braquial que la del pectoral mayor con apoyo a la anchura de hombros.. No obstante, constataron los mayores registros EMG para ambos músculos cuando analizaban el agarre de tipo estrecho durante la ejecución del gesto. Afirman los autores, que un alto registro electromiográfico no es reflejo de alta tensión muscular, ya que ante una misma carga, si realizamos un movimiento concéntrico a mayor velocidad, el reclutamiento neuromuscular es también mayor y sin embargo, la tensión muscular generada es menor. No obstante, siguiendo los preceptos de la caída libre de los cuerpos de Galileo, tanto mayor sea la velocidad ascensional de un cuerpo, mayor es la altura. Por ello, podemos seguir afirmando la existencia de correlación positiva entre los registros EMG y los datos de tiempo de vuelo y altura del Ergojump. Independientemente de la participación mioeléctrica de ambos músculos en el gesto, y basándonos en los hallazgos de Murr (1997) (10), analizó la fiabilidad y objetividad de dos test que incluían la flexo-extensión de codos, como son el Fitnessgram y el Test Militar sobre $\quad 100 \quad$ estudiantes universitarios, concluyendo que ambos test, son válidos y fiables para medir la fuerza y resistencia de brazos y hombros.

Evidentemente, en virtud del porcentaje de peso corporal que recae sobre las manos en el gesto según Gouvali et al. (2005) (13), que es de un $66.4 \%$ en la posición normal, mientras que con apoyo de rodillas es de un $52.9 \%$, entendemos que la máxima capacidad de realizar repeticiones no es reflejo de la fuerza absoluta de las extremidades superiores de un individuo dado que la carga no es máxima. Mayhew et al. (1991) (5), constataron cómo el gesto de flexo-extensión de brazos no es predictor de $1 \mathrm{RM}$ en press de banca, y por tanto muestran que el gesto objeto de estudio no son un reflejo de la fuerza máxima de las extremidades superiores.

Contrariamente, Invergo et al. (1991) (7), hallaron que el gesto en cuestión, en algunos casos puede ser una alternativa viable a la clásica 1RM en press de banca, para la determinación de la máxima capacidad de levantamiento de peso de un individuo.

Rev. Ib. CC. Act.Fis. Dep. 
Según nuestros hallazgos de investigación, nos encontramos en consonancia con Pate et al. (1993) (6) quienes investigaron la validez de cinco tests de campo para la valoración de la fuerza-resistencia en niños de 9-10 años. Entre ellos, utilizaron el de flexo-extensiones de brazos, observando que si bien, todos ellos no eran válidos para la medición de la fuerza máxima y tampoco de la fuerza-resistencia, pero sí poseían validez de constructo para ser utilizados como medidas de fuerza muscular relativa al peso corporal individual.

\section{CONCLUSIONES}

Hemos demostrado de un modo fiable la existencia de correlación positiva entre los registros del electromiógrafo y de la Plataforma de Bosco (Tabla 6); sin embargo, no ha sido posible establecer una generalización para todo tipo de población puesto que hubiese hecho falta mayor número de sujetos, no en el caso del primer instrumento, pero sí en el del segundo.

TABLA 6: Resultados del análisis de correlación intraclase con la aplicación GT

\begin{tabular}{cc}
\hline MODELOS & COEFICIENTE DE CORRELACIÓN \\
\hline $\mathrm{d} / \mathrm{xt}$ & $\mathrm{e} 2=1.000$ \\
$\mathrm{~d} / \mathrm{xh}$ & $\mathrm{e} 2=1.000$ \\
$\mathrm{x} / \mathrm{dt}$ & $\mathrm{e} 2=1.000$ \\
$\mathrm{x} / \mathrm{dh}$ & $\mathrm{e} 2=1.000$
\end{tabular}

(d=EMG medios, $\mathrm{x}=\mathrm{EMG}$ máximos, $\mathrm{t}=$ tiempos de vuelo, $\mathrm{h}=$ alturas)

Como se expone en la Tabla 7 , sobre estimaciones de los distintos planes de medida, con los 50 sujetos que han realizado el experimento podríamos generalizar los datos referentes al electromiógrafo, pero no los propios de la plataforma.

TABLA 7: Resultados del análisis de Generalizabilidad ( $($ ) y fiabilidad (e2) de los distintos planes de medida de facetas según distintas variables dependientes.

\begin{tabular}{lcccc}
\hline $\begin{array}{l}\text { Variable } \\
\text { Dependiente }\end{array}$ & $\begin{array}{c}\text { Modelo de } \\
\text { Facetas } \\
\text { MLE/S }\end{array}$ & $\begin{array}{c}\text { Modelo de } \\
\text { Facetas } \\
\text { SLE/M }\end{array}$ & $\begin{array}{c}\text { Modelo de } \\
\text { Facetas } \\
\text { SEM/L }\end{array}$ & $\begin{array}{c}\text { Modelo de } \\
\text { Facetas } \\
\text { SLM/E }\end{array}$ \\
\hline \multirow{2}{*}{ EMG Máximos $(\mathrm{x})$} & $\mathrm{e} 2=0.98^{*}$ & $\mathrm{e} 2=0.10$ & $\mathrm{e} 2=0.82^{* *}$ & $\mathrm{e} 2=0.93^{*}$ \\
& $\Phi=0.98^{*}$ & $\Phi=0.05$ & $\Phi=0.81^{* *}$ & $\Phi=0.93^{*}$ \\
EMG Medias (d) & $\mathrm{e} 2=0.98^{*}$ & $\mathrm{e} 2=0.07$ & $\mathrm{e} 2=0.82$ & $\mathrm{e} 2=0.94^{*}$ \\
& $\Phi=0.98^{*}$ & $\Phi=0.04$ & $\Phi=0.81$ & $\Phi=0.94^{*}$ \\
Alturas (h) & $\mathrm{e} 2=0.78$ & $\mathrm{e} 2=0.98^{*}$ & $\mathrm{e} 2=0.78^{* *}$ & $\mathrm{e} 2=0.81^{* *}$ \\
& $\Phi=0.59$ & $\Phi=0.98^{*}$ & $\Phi=0.78^{* *}$ & $\Phi=0.80^{* *}$ \\
Tiempos de vuelo $(\mathrm{t})$ & $\mathrm{e} 2=0.77$ & $\mathrm{e} 2=0.99^{*}$ & $\mathrm{e} 2=0.77$ & $\mathrm{e} 2=0.79^{* *}$ \\
& $\Phi=0.56$ & $\Phi=0.99^{*}$ & $\Phi=0.77$ & $\Phi=0.78^{* *}$ \\
\hline
\end{tabular}

La causa puede deberse a la variabilidad de las mediciones durante el desarrollo de la investigación, con respecto a la primera estimación inicial con cuatro sujetos.
Cierto es, que los rangos de edad de las muestras han sido bastante amplios, concretamente entre $14 \mathrm{y}$ 53 años, lo cual ha podido ser un factor que pudiese haber influido en la homogeneidad de la medida,

Rev. Ib. CL. Act.Fis. Dep. 
suponiendo por tanto la imposibilidad de generalizar los resultados con las 50 muestras previstas. En los prolegómenos de la investigación, consideramos que si queríamos establecer una generalización de los resultados, la muestra debía ser diversa Podemos afirmar que los sujetos han obtenido mayores registros electromiográficos, cuanto mayores han sido los tiempos de vuelo, y por tanto, gracias a la correlación existente, se puede estimar y comparar la capacidad de impulsión de las extremidades superiores.

Evidentemente, la fuerza generada por el sujeto experimental en cuestión, estará condicionada por la magnitud de la carga del peso corporal propio del individuo que se somete a la prueba, por ello, hemos de hablar de mediciones de fuerza relativa. Ésta es la razón por la cual, los resultados obtenidos en el Ergojump no pueden ser comparados entre sujetos, pero sí resultan ser mediciones fiables en

\section{REFERENCIAS BIBLIOGRÁFICAS} 1. COGLEY, R.M., TEASHA, A., ARCHAMBAULT, J.F., MANDY, M., KOVERMAN, J.W., YOUDAS, J.W., and HOLLMAN, J.H. (2005). Comparison of Muscle Activation Using Various Hand Positions During the Push-up exercise.

2. LAFOND, D. (2003). Contribution a l'evaluation de l'equilibre quasi-statique a l'aide d'una plate-forme de force. Tesis de Doctorado. Universidad de Montreal

3. MARQUARDT, B.P. (1996). Force plate validation and reliability: $A$ study of forces in the $x, y$ and $z$ axes. Tesis de Doctorado. D'Youville College. Universidad de Nueva York.

4. SANDERS, S.C.(1996). Validation of a piezoelectric force plate portable unit: $C O P$ estimation. Tesis de Doctorado. D'Youville College. Universidad de Nueva York.

5. MAYHEW, J.L., BALL, T.E., ARNOLD, M.D., and BOWER, J.C. (1991). Push-ups condiciones intrasujetos. Así, podríamos utilizar la plataforma como un medio de control del entrenamiento de la fuerza intrasujeto de un modo válido y fiable.

Se abre pues, un campo amplio de posibles líneas de investigación en el que resultaría interesante:

1. Ampliar la muestra de sujetos de un modo suficiente para generalizar la correlación. (Según el nuevo plan de estimación, en torno a 320 sujetos).

2. Una vez conseguido el anterior, centrarse en muestras de deportistas según la disciplina practicada.

3. Comprobar si al igual que ocurre con las extremidades inferiores, podemos establecer una correlación positiva entre alturas y tiempos de vuelo, con velocidad y frecuencia de movimientos de las extremidades superiores en gestos propios de deportes como son por ejemplo la natación.

As a Measure of Upper Body Strength. The Journal of Strength and Conditioning Research, 5 (1), 16-21.

6. PATE, R.R., BURGESS, M.L., WOODS, J.A., ROSS, J.G., BAUMGARTNER, T. (1993). Validity of field tests of upper body muscular strength. Research Quarterly for Exercise and Sport, 64(1), 17-24.

7. INVERGO, J.J., BALL, T.E. and LOONEY, M. (1991). Relationship of Pushups and Absolute Muscular Endurance to Bench Press Strength. The Journal of Strength and Conditioning Research, 5 (3), 121-125.

8. BENTZ, R.J., M.S. (2003). The effects of eight weeks of manual resístance push-ups on chest strength measures among collegiate students. Dissertation Abstracts AAT 1414919. (Southern Connecticut State University, 40 pgs.).

9. BOWERSOCK, A.E. (1999). The anthropometric influence and comparative

Rev. Ib. Cᄃ. Act.Fis. Dep. 
work output in push up performance. Dissertation Abstracts AAT 9932776. (University of Arkansas, 115pgs.).

10. MURR, M. S. (1997). Objectivity and reliability of two push-up test protocols for male college students. . Dissertation Abstracts AAT 9817866 (University of Georgia, 97 pgs.).

11. HALEM, D. (2002). Pump up with pushups. Joe Weider's Muscle \& Fitness, 63 (2), pg. 116

12. HRYSOMALLIS, C., KIDGELL, D. (2001). Effect of heavy dynamic resistive exercise on acute upper-body power. The Journal of Strength and Conditioning Research, 15 (4), 426-30.

13. GOUVALI, M.K., and BOUDOLOS, K. (2005). Dynamic and Electromyographical Analysis in Variants of Push-Up Exercise. The Journal of Strength and Conditioning Research, 19 (1), 146-151.

14. LEAR, L.J., GROSS, M.T. (1998). An electromyographical analysis of the scapular stabilizing synergists during a push-up progression. The Journal of Orthopedic and Sports Physical Therapy, 28 (3), 146-57.

15. DONKERS, M.J., An, K.N., CHAO, E.Y., MORREY, B.F. (1993). Hand position affects elbow joint load during push-up exercise. Journal of Biomechanics, 26(6), 625-32.

16. LEHMAN, G.J. (2005). The influence of grip width and forearm pronation/supination on upper-body myoelectric activity during the flat bench press. The Journal of Strength and Conditioning Research, 19 (3), 587-91. 17. CASTELLANO, J. (2000). Observación y análisis de la acción de juego en fútbol. Tesis Doctoral: Universidad del País Vasco. 18. BLANCO, A. (2001). Generalizabilidad de observaciones uni y multifaceta: estimadores LS y ML. Metodología de las Ciencias del Comportamiento, 3(2), 161193.

19. BLANCO, A., CASTELLANO, J. and HERNÁNDEZ, A. (2000). Generalizabilidad de las observaciones de la acción del juego en el fútbol. Psicothema, 12, 2, 81-86.

The Journal of Strength and Conditioning Research, 19 (3), 628-633.

20. MOLINERO, L. M. (2001). Errores de medida en variables numéricas: Correlación y Concordancia. SEH: Asociación de la Sociedad Española de Hipertensión.

Rev. Ib. Cᄃ. Act.Fis. Dep.
21. PELEGRINA, M. (1999). La investigación experimental en psicología. España: Aljibe.

22. HERNÁNDEZ, R., FERNANDEZ, C., BAPTISTA, P. (2000). Metodología de la investigación. (2ª Edición). México: Mc Graw Hill. 